\title{
Bacteriemias e infección endovascular por Campylobacter spp: nuestra experiencia en un cuarto de siglo de historia
}

\author{
Alberto Fica C., Lorena Porte T., Stephanie Braun J., Nicolás Veas P., \\ Claudia Pavez A., Jeannette Dabanch P. y Ricardo Morales I.
}

\section{Bacteremia and endarteritis cases secondary to Campylobacter spp. in a metropolitan hospital. Our experience along a quarter of a century}

Six cases of bacteremia and one of endarteritis were identified between 1986 and 2010 in a general hospital in Chile. Five of these cases occurred during the second half of this timeframe, Campylobacter fetus predominated (5 out of 7) and the majority of the infections presented during warmer months. The mean age was 32.4 years (range 19 to 63) all had comorbidities, and main clinical manifestations included fever with diarrhea. Four patients developed hypotension and two septic shock. The latter, associated to C. fetus bacteremia, died before microbiological diagnosis. Six out of 7 patients received antimicrobial therapy. During 2004 and 2010, the rates of Campylobacter spp. positive stool cultures in the same hospital increased 4 times, suggesting an emerging profile. Bacteremia and endarteritis by Campylobacter spp. can develop in vulnerable patients and manifest as fever with or without diarrhea. Finding curved or spiral shaped gram negative rods in blood cultures leads to suspect this pathogen. Species identification is of utmost importance due to antimicrobial resistance especially in C. jejuni. Prognosis is unfavorable due to host characteristics, and case-fatality rate is high.

Key words: Campylobacter fetus, bacteremia, endarteritis.

Palabras clave: Campylobacter fetus, bacteriemia, endarteritis.

\section{Introducción}

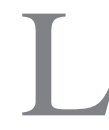

as bacteriemias causadas por Campylobacter spp., aunque infrecuentes, se asocian a pacientes con enfermedades debilitantes y en ocasiones pueden ser letales ${ }^{1,2}$. Las infecciones extra-intestinales por este agente son de reciente aparición y reporte en Chile ${ }^{3}$. Con el fin de conocer las características clínicas y la evolución de este tipo de infecciones se presenta una serie de casos de bacteriemias y un caso de infección endovascular registrados en nuestra institución. Además, se incluye un análisis de los coprocultivos positivos con este agente para determinar una posible emergencia o expansión del microorganismo en los últimos años.

\section{Pacientes y Métodos}

Casos clínicos. Los casos fueron identificados a través del registro de bacteriemias del Laboratorio Clínico del Hospital Militar de Santiago. Se incluyeron pacientes mayores de 15 años en el período comprendido entre enero de 1986 y junio de 2010. El análisis se efectuó mediante la revisión de las fichas clínicas de los pacientes o en base a información parcial disponible. Los datos se presentan se acuerdo a las características clínicas, co-morbilidad, tratamiento y evolución. Los resultados se presentan en tablas sumatorias.

Hemocultivos. Hasta el año 1996 (julio) se aplicaba un sistema manual de hemocultivos; desde ese año (agosto), hasta abril de 2009, se utilizó un sistema de hemocultivos automatizados (Bact T Alert ${ }^{\circledR}$, BioMerieux, Francia) y desde mayo de 2009, el sistema automatizado Versatrek ${ }^{\circledR}$ (Trek Diagnostic Systems, USA).

Progresión de Campylobacter como agente de cuadros diarreicos. Se analizó la proporción de cultivos positivos a Campylobacter spp., identificados en el laboratorio clínico del mismo hospital desde enero de 2004 hasta junio de 2010 y su tendencia en el tiempo. El análisis estadístico se realizó mediante el coeficiente de correlación de Pearson y la prueba de diferencia de proporciones.

\author{
Hospital Militar de Santiago, \\ Chile. \\ Departamento de Medicina \\ Servicio de Infectología (AFC, NVP, \\ (PA, JDP, RMI). \\ Laboratorio de Microbiología \\ (LPT, SBJ). \\ Conflicto de interés: ninguno. \\ Financiamiento: ninguno.
}

Recibido: 10 de septiembre 2010 Aceptado: 9 de marzo de 2011

Correspondencia a: Alberto Fica Cubillos albertofica@gmail.com 


\section{Descripción de los casos}

En el período estudiado se identificaron seis casos de bacteriemias en pacientes $>15$ años y un caso de infección vascular, asociados a diferentes especies del género Campylobacter (Tabla 1). La información disponible no permitió acceder a datos sobre los hábitos de alimentación de los pacientes, las alteraciones de laboratorio ni la respuesta a la terapia antimicrobiana. Los aislados no fueron sometidos a estudio de susceptibilidad antimicrobiana.

Se observó una concentración de casos en la segunda mitad del período de 25 años de esta serie (5/7; 71,4\%) y una agrupación de las bacteriemias en los meses más calurosos del año (5 de 7; 71,4\%). Además, los siete pacientes presentaron diversas co-morbilidades, exceptuando inmunosupresiones habituales (Tabla 1 ) y el predominio de $C$. fetus como agente de su cuadro clínico. Cuatro pacientes eran de sexo masculino y la edad promedio fue de 32,4 años (rango 19 a 63 años).

Diarrea y fiebre, presentes en cinco de siete pacien- tes, fueron las manifestaciones clínicas más frecuentes $(71,4 \%)$. Cuatro de siete pacientes presentaron hipotensión arterial $(57,1 \%)$ y dos pacientes presentaron shock séptico (28,6\%). Una mujer evolucionó con fiebre y artralgias, otro caso con descompensación de una cirrosis hepática y encefalopatía y uno como neutropenia febril con diarrea. El dolor abdominal fue infrecuente en esta serie (Tabla 1). El paciente sin bacteriemia debutó con un cuadro de trombosis de un aneurisma de la aorta abdominal, con dolor durante 15 días, sin fiebre, hipotensión arterial o diarrea (caso 7). Durante el acto quirúrgico se identificó una zona inflamatoria perivascular que se envió a cultivo.

Tratamiento y evolución. Seis de los siete pacientes $(85,7 \%)$ recibieron tratamiento antimicrobiano. Sin embargo, en sólo dos de ellos se adecuó el esquema terapéutico luego de conocerse la etiología. En ambos casos se agregaron macrólidos (casos 2 y 3), obteniéndose una respuesta favorable. En otros dos pacientes, el tratamiento

\begin{tabular}{|c|c|c|c|c|c|c|c|}
\hline Variable & Caso 1 & Caso 2 & Caso 3 & Caso 4 & Caso 5 & Caso 6 & Caso 7 \\
\hline Edad (años) / Sexo & $33 \mathrm{~F}$ & $21 \mathrm{M}$ & $19 \mathrm{~F}$ & $49 \mathrm{M}$ & $42 \mathrm{~F}$ & $63 \mathrm{M}$ & $73 \mathrm{M}$ \\
\hline Año de ocurrencia & 1986 & 1991 & 2002 & 2003 & 2008 & 2010 & 2007 \\
\hline Mes & Marzo & Febrero & Agosto & Noviembre & Abril & Junio & Noviembre \\
\hline Co-morbilidad* & Talasemia menor & LLA en remisión & $\begin{array}{l}\text { Malformaciones } \\
\text { venosas por telan- } \\
\text { gectasia heredita- } \\
\text { ria familiar }\end{array}$ & $\begin{array}{l}\text { Cardiopatía coro- } \\
\text { naria, tabaquismo, } \\
\text { dislipidemia }\end{array}$ & $\begin{array}{l}\text { Hipertensión } \\
\text { pulmonar }\end{array}$ & $\begin{array}{l}\text { Cirrosis hepática } \\
\text { Child Pugh B por } \\
\text { virus B y alcohol }\end{array}$ & $\begin{array}{l}\text { HTA, cáncer } \\
\text { prostático en } \\
\text { tratamiento, } \\
\text { tabaquismo }\end{array}$ \\
\hline Especie & C. fetus & C. jejuni & C. fetus & C. fetus & C. fetus & C. jejuni & C. fetus \\
\hline Cuadro clínico & S febril + artralgias & $\begin{array}{l}\text { Neutropenia febril } \\
+ \text { dolor abdominal } \\
+ \text { diarrea }\end{array}$ & S febril + diarrea & $\begin{array}{l}\text { Diarrea + vómitos } \\
\text { + shock séptico }\end{array}$ & $\begin{array}{l}\text { Diarrea + shock } \\
\text { séptico }\end{array}$ & $\begin{array}{l}\text { Descompensación } \\
\text { de encefalopatía }\end{array}$ & $\begin{array}{l}\text { Dolor abdominal } \\
\text { por trombosis con } \\
\text { aneurisma de aorta } \\
\text { iliaca derecha }\end{array}$ \\
\hline Fiebre* & Sí & Sí & Sí & ¿? & ¿? & No & No \\
\hline Hipotensión arterial & No & No & Sí & Sí & Sí & Sí & No \\
\hline Shock & No & No & No & Sí & Sí & No & No \\
\hline Diarrea & No & Sí & Sí & Sí & Sí & Sí & No \\
\hline Dolor abdominal & No & Sí & No & ¿? & No & No & Sí \\
\hline Diagnóstico & $\begin{array}{l}\text { Formas espirilares } \\
\text { en hemocultivos. } \\
\text { Diagnóstico post } \\
\text { egreso }\end{array}$ & Hemocultivo & Hemocultivo & Hemocultivo & $\begin{array}{l}\text { Hemocultivo con } \\
\text { identificación post } \\
\text { mortem }\end{array}$ & Hemocultivo & $\begin{array}{l}\text { Cultivo de tejido } \\
\text { perivascular }\end{array}$ \\
\hline $\begin{array}{l}\text { Tratamiento } \\
\text { antimicrobiano }\end{array}$ & Sin tratamiento & $\begin{array}{l}\text { Ceftazidima }+ \\
\text { amikacina }+ \\
\text { eritromicina oral }\end{array}$ & $\begin{array}{l}\text { Ceftriaxona }+ \\
\text { azitromicina oral }\end{array}$ & $\begin{array}{l}\text { Ciprofloxacina }+ \\
\text { metronidazol }\end{array}$ & $\begin{array}{l}\text { Ceftriaxona }+ \\
\text { metronidazol }\end{array}$ & Ceftriaxona & $\begin{array}{l}\text { Imipenem + van- } \\
\text { comicina por fiebre } \\
\text { post operatoria }\end{array}$ \\
\hline Evolución & Favorable & Favorable & Favorable & Fallece & $\begin{array}{l}\text { Fallece a las } 48 \\
\text { horas }\end{array}$ & Favorable & Favorable \\
\hline
\end{tabular}




\section{Experiencia Clínica}

se dirigió hacia la sospecha de peritonitis bacteriana espontánea (caso 6) y a un cuadro febril postoperatorio (paciente portador del aneurisma trombosado) (caso 7). Finalmente, otros dos pacientes fallecieron pocos días después del ingreso sin conocerse el agente etiológico, por lo que recibieron un esquema de tratamiento según su presentación clínica al ingreso (casos 4 y 5). La paciente que no recibió tratamiento presentó una evolución favorable (caso 1). La resolución fue favorable en cinco de los siete casos (71,4\%). Los dos pacientes que presentaron shock fallecieron (28,6\%), aislándose C. fetus.

Emergencia de Campylobacter como agente de diarrea. El porcentaje de coprocultivos positivos a Campylobacter spp., aumentó progresivamente en nuestra institución entre los años 2004 y 2010 (Figura 1), registrándose un aumento aproximado de cuatro veces (desde $<0,5 \%$ a $>2 \%)(\mathrm{p}<0,0005$ por prueba de diferencia de proporciones). El coeficiente de correlación de Pearson fue de 0,89 .

\section{Discusión}

El aumento reciente de los coprocultivos positivos a Campylobacter procesados en el laboratorio clínico institucional sugiere que este agente se estaría comportando como un patógeno emergente. Aunque los datos son escasos y no cuentan con una base poblacional que demuestre un cambio en la incidencia, este comportamiento sería similar al registrado con otros patógenos emergentes ligados a la centralización de la industria alimentaria en Chile. Este fenómeno fue descrito en Escherichia coli enterohemorrágica y Salmonella enterica serotipo Enteritidis en la década de 1990 y en Listeria monocytogenes en años más recientes ${ }^{4-10}$. La posible emergencia de Campylobacter y su expresión como causa marginal de infección extra-intestinal queda registrada por la concentración de casos en el último período y por la primera descripción de un caso de bacteriemia en Chile en los últimos años. ${ }^{3}$ En países industrializados, las bacteriemias por Campylobacter se expresan en cifras endémicas o incluso regresivas ${ }^{2,11,12}$. El aumento de casos en nuestra institución no parece depender de un cambio de los sistemas de hemocultivos, por cuanto bajo el mismo sistema automatizado instalado el año 1996 sólo se comenzaron a detectar casos desde el año 2000, habiéndose registrado casos remotos en 1986 y 1991, pesquisados con un sistema manual de hemocultivos.

Los integrantes del género Campylobacter están involucrados en cuadros de zoonosis. La transmisión de este patógeno se asocia al consumo de carne inadecuadamente cocida, consumo de leche no pasteurizada, agua contaminada o al contacto con mascotas, y se estima que

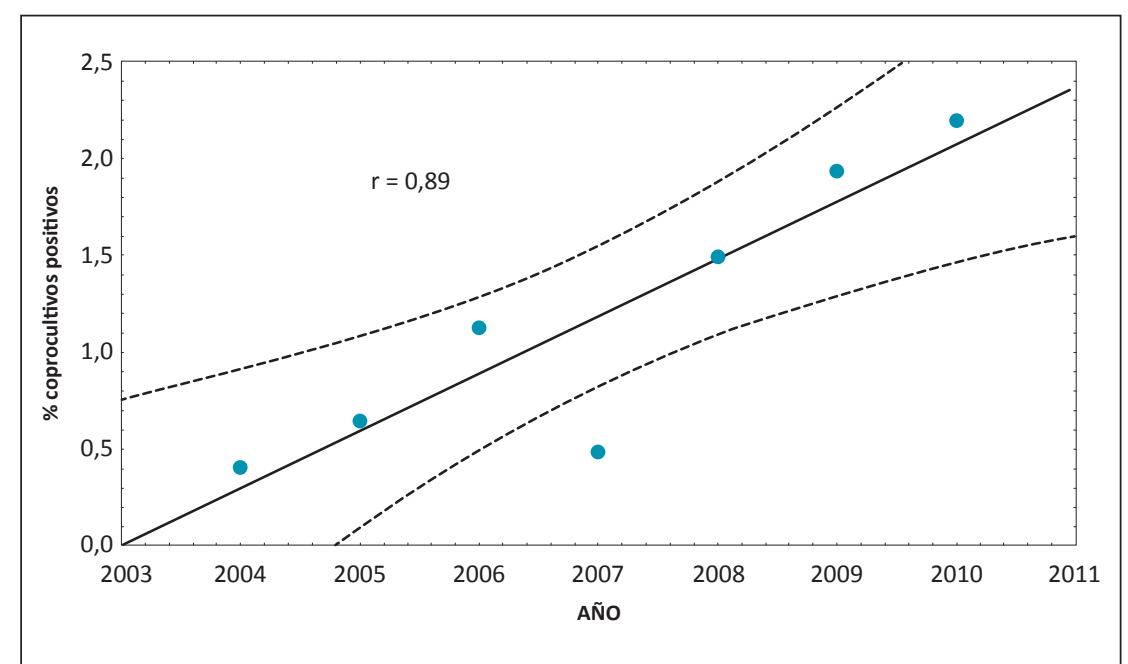

Figura 1. Tasa de coprocultivos positivos a Campylobacter spp. en el Hospital Militar de Santiago (desde 2003 a junio de 2010).

al menos $80 \%$ de los casos de infecciones por este género son adquiridos por alimentos, con una tasa de hospitalización de $10 \%$ y una letalidad de 1 por $1.000 \operatorname{casos}^{13}$. Al igual que en el caso de Salmonella Enteritidis, la mayor parte de las infecciones por Campylobacter aparecen ligadas a productos avícolas. En Chile, Figueroa y cols., demostraron el extenso grado de contaminación de las carcasas de aves y de superficies y sitios de trabajo por Campylobacter spp., en dos plantas faenadoras en la Región Metropolitana, en los años 2006-2007. Las cifras de contaminación en algunas etapas del proceso de faenado fueron cercanas a $90 \%{ }^{14}$.

Epidemiología y clínica. Históricamente se ha planteado que $C$. fetus se asocia a cuadros de bacteriemia y rara vez a casos de enteritis y que en contraste, $C$. jejuni se asocia a cuadros de enteritis y escasamente a pacientes con bacteriemia ${ }^{1}$. Sin embargo, la evidencia indica que los casos de bacteriemia por diferentes especies de Campylobacter ocurrirían como un evento marginal (en pacientes vulnerables) respecto al total de infecciones intestinales, según la especie epidemiológica predominante en un entorno geográfico. Es así como C. fetus predomina como agente de bacteriemia en Francia y Canadá, y C. jejuni en España y Dinamarca ${ }^{11,12,15,16}$.

Los grupos vulnerables a bacteriemias por este género incluyen, entre otros, a pacientes mayores, a los afectados por diabetes mellitus, cirrosis hepática o alcoholismo, cirugía gastrointestinal, neoplasias onco-hematológicas, infección por VIH y trasplante de órganos sólidos/ precursores hematopoyéticos o inmunosupresión por corticoesteroides. ${ }^{12,15,16}$ Algunas de estas situaciones fueron detectadas en nuestra serie. Otros autores describen como 
factores predisponentes a las patologías cardiovasculares y pulmonares crónicas, como ocurrió también en algunos casos $^{11,12}$.

La presentación clínica observada es concordante con lo descrito en la literatura científica, donde la fiebre con manifestaciones digestivas -hechos predominantes en esta serie- o extra-digestivas, representa el perfil más característico. Las bacteriemias por $C$. jejuni se asocian más frecuentemente a diarrea que las observadas por C. fetus ${ }^{11,16}$. Las bacteriemias por este género patógeno son muy infrecuentes en cualquier serie publicada y representan una muy baja fracción respecto al total de egresos, hemocultivos o como incidencia por habitantes/ cada año ${ }^{11,12,16}$. Los seis casos presentados en esta serie, junto al evento de infección vascular, concuerdan con la baja casuística registrada anualmente.

La infección vascular, con o sin bacteriemia, representa una más de las manifestaciones dentro de la diversidad de cuadros clínicos asociados a este patógeno y se asume ligada al tropismo vascular, especialmente de C. fetus $^{11,15}$. Las infecciones vasculares incluyen casos de endocarditis, infección de prótesis vasculares o de un aneurisma abdominal, como fue uno de los pacientes descritos en esta serie ${ }^{11,12}$. También puede considerarse como tal la infección observada en la paciente con una telangectasia hereditaria familiar.

Las infecciones extra-intestinales por Campylobacter spp., parecen estar más relacionadas con la vulnerabilidad del hospedero que con la presencia de factores de virulencia, tal como lo señala Nielsen en su estudio. Este autor no pudo demostrar diferencias en las características de los aislados de $C$. jejuni obtenidos de hemocultivos y los presentes en coprocultivos ${ }^{17}$. Entre los factores de virulencia de $C$. fetus destaca la capacidad de resistir el efecto bactericida del suero. De esta manera, la probabilidad de desarrollar bacteriemia y no solamente una infección o portación intestinal depende de las características del hospedero haciendo que las infecciones del torrente sanguíneo se presenten como "la punta del iceberg” de la circulación de Campylobacter spp en la comunidad ${ }^{12,13}$.

Tratamiento y evolución. El tratamiento de las bacteriemias por Campylobacter spp enfrenta varios problemas que incluyen, entre otros aspectos, la naturaleza del hospedero afectado y problemas de resistencia a diferentes compuestos antimicrobianos. En Chile, al igual que en otras partes del mundo se han reportado porcentajes crecientes de resistencia a fluoroquinolonas, un hecho que impide recomendar tratamientos empíricos basados en este compuesto. Al respecto, García y cols, indican cifras > $30 \%$ de resistencia a quinolonas en aislados de C. jejuni ${ }^{18}$. La resistencia a quinolonas es un fenómeno universal y afecta a todas las especies de importancia clínica pertenecientes a este género ${ }^{19,20}$. La susceptibilidad a $\beta$-lactámicos parece depender del compuesto específico y de la especie analizada, siendo más complejo el problema en C. jejuni. En esta especie, los compuestos con mejor actividad corresponden a aquellos que tienen mayor afinidad por las PBPs, que son de un tamaño relativamente pequeño, y que tienen una carga iónica dipolar que les permite penetrar mejor a través de las porinas. En este grupo se encuentran imipenem, amoxicilina y las cefalosporinas de cuarta generación como cefepime y cefpirome ${ }^{21}$. Estos mismos fenómenos explican las limitaciones de la actividad in vitro de ampicilina, cefalosporinas de primera a tercera generación y piperacilina contra $C$. jejuni y además, para algunos compuestos, su asociación con un mayor riesgo de muerte (ver más adelante) ${ }^{19,21}$. El ácido clavulánico es el compuesto inhibidor de $\beta$-lactamasas que tiene un mejor perfil de inhibición y penetración en esta bacteria pero sólo es de utilidad cuando acompaña a uno de los compuestos con actividad mencionados anteriormente ${ }^{21}$. Estos antecedentes indican que es importante contar con la identificación de la especie bacteriana. Imipenem y gentamicina ofrecen una excelente actividad in vitro ${ }^{21}$. En contraste a $C$. jejuni, la especie $C$. fetus no presenta los problemas de resistencia descritos para cefalosporinas de tercera generación ${ }^{15}$. La susceptibilidad a macrólidos es variable en ambas especies pero poco aplicable al manejo de bacteriemias ${ }^{15,18,19}$. En esta serie, su uso por vía oral fue complementario a otros esquemas.

La duración del tratamiento comunicada en la literatura médica ha sido variable $y$, dado el riesgo de recaída, se acostumbra utilizar tratamientos prolongados de cuatro o más semanas, inicialmente por vía parenteral. Debido a la baja frecuencia de esta patología, no se cuenta con ensayos randomizados que permitan determinar el mejor esquema terapéutico.

Los casos letales en esta serie se asociaron a la presencia de shock al inicio y sólo en caso de infecciones por $C$. fetus. La aparición de cuadros de shock séptico al inicio de la infección o durante las recaídas ha sido descrita por varios autores ${ }^{11,16}$. A pesar de que la mortalidad cruda reportada en algunas series ha sido nula o muy baja (0-4\%), en otras series ésta ha alcanzado las altas cifras observadas en nuestros pacientes ( $\sim 30 \%$ ) ya fuese asociadas a $C$. fetus o a $C$. jejuni ${ }^{11,12,16,22}$. Sólo un tercio de los decesos ha sido atribuido directamente a la infección ${ }^{16}$. En un análisis multivariado, los factores asociados a un desenlace fatal fueron distintos para las infecciones asociadas a C. fetus respecto a las provocadas por $C$. jejuni. En el primer caso, el fallecimiento al mes de evolución aparece ligado a la existencia de una neoplasia o a la fiebre sin un foco de origen. Este último fenómeno estuvo presente en nuestra serie en los casos letales. En el caso de infecciones por otras especies, como C. jejuni, los factores relacionados con letalidad correspondieron a problemas terapéuticos, como terapia empírica inapropiada o el uso de cefalos- 
porinas de tercera generación ${ }^{19}$. En un paciente portador de talasemia menor, una condición no reconocida como predisponente a bacteriemias por este patógeno, la infección evolucionó en forma autolimitada y sin tratamiento antimicrobiano, reforzando la importancia del hospedero en el pronóstico de la enfermedad.

Limitaciones. Los datos presentados en esta serie no están completos debido a fallas en los registros clínicos y a su naturaleza retrospectiva. Tampoco se contó con estudios de susceptibilidad antimicrobiana en las cepas aisladas. Del mismo modo, la posible emergencia de Campylobacter spp., como agente de bacteriemias o infecciones endovasculares, no está expresada como una tasa poblacional y, por lo tanto, los cambios en su incidencia son especulativos. Campylobacter jejuni ha sido descrito como agente de diarrea en lactantes en Chile desde que se inició su búsqueda en la década de 1980, curiosamente en forma coincidente con el primer caso de bacteriemia en esta serie en esa década ${ }^{23}$.

Conclusiones. Las bacteriemias o infecciones vasculares por Campylobacter spp., aunque infrecuentes, pueden presentarse en una diversidad de pacientes vulnerables y debutar como cuadros febriles, en presencia o ausencia de diarrea. La sospecha se establece por el hallazgo de bacilos gran negativos curvo-espirilares en sangre y la identificación de la especie involucrada es de suma importancia debido a la escasa actividad terapéutica de cefalosporinas de tercera generación y quinolonas. El pronóstico de estas bacteriemias es grave debido a las características del hospedero y a su elevada letalidad.

\section{Resumen}

Se presentan seis casos de bacteriemia y uno de infección vascular por Campylobacter spp, observados en 25 años, con el fin de describir sus características clínicas. Cinco de ellos se registraron en la segunda mitad del período, en concomitancia con el incremento de este agente en el porcentaje de coprocultivos, lo que sugiere un perfil emergente. Las infecciones fueron más frecuentes en los meses cálidos, asociadas principalmente a C. fetus (5 de 7) y a co-morbilidad. La edad promedio de los pacientes fue de 32,4 años (rango 19 a 63 años) y todos tenían comorbilidades. Las manifestaciones clínicas más frecuentes fueron diarrea y fiebre (5 de 7 casos) y dos pacientes cursaron con shock séptico (28,6\%). La evolución fue favorable en cinco pacientes pero los dos que presentaron shock asociado a $C$. fetus fallecieron (28,6\%). Las bacteriemias o infecciones vasculares por Campylobacter spp., aunque infrecuentes, pueden presentarse en pacientes vulnerables y debutar como cuadros febriles, en presencia o ausencia de diarrea. La identificación de la especie involucrada es de suma importancia debido a la escasa actividad terapéutica de cefalosporinas de tercera generación y quinolonas. El pronóstico de estas bacteriemias es grave debido a las características del hospedero y a su elevada letalidad.

\section{Referencias}

1.- Bacilos gramnegativos curvos y fermentadores, oxidasa positivos: campilobacterias y vibriones. En: Winn WC, Allen SD, Janda WM, Koneman EW, Procop GW, Schreckenberg PC, Woods GL. (Eds). Koneman, Diagnóstico Microbiológico, Texto y Atlas en Color. Editorial Médica Panamericana. Buenos Aires. $6^{\text {a }}$ Edición. 2008; pp. 372-406.

2.- Allos N M. Campylobacter jejuni infections: Update on emerging issues and trends. Clin Infect Dis. 2001; 32: 1201-6.

3.- Fica A, Illanes V, Sakurada A, Vidal M, Valenzuela ME. Bacteriemia por Campylobacter fetus subsp fetus en un paciente inmunosuprimido. Rev Chil Infectol 2006; 23: 336-9.

4.- Ríos M, Prado V, Trucksis M, Arellano C, Borie C, Alexandre M, et al. Clonal diversity of Chilean isolates of enterohemorrhagic Escherichia coli from patients with hemolyticuremic syndrome, asymptomatic subjects, animal reservoirs, and food products. J Clin Microbiol 1999; 37: 778-81.

5.- Fernández J, Fica A, Ebensperger G,
Calfullan H, Prat S, Fernández A, et al. Molecular epidemiology of Chilean Salmonella enterica serotype Enteritidis isolates by pulsed-field gel electophoresis and bacteriophage typing. J Clin Microbiol 2003; 41 (4): 1617-22.

6.- $\quad$ Prat S, Fernández A, Fica A, Fernández J, Alexandre M, Heitmann I. Tipificación fágica de aislados de Salmonella enteritidis de muestras clínicas, alimentarias y avícolas en Chile. Rev Panam de Salud Pública-Pan Am J Public Health 2001; 9: 7-12.

7.- $\quad$ Fica A, Alexandre M, Prat S, Fernández A, Fernández J, Heitmann I. Cambios epidemiológicos en salmonelosis. Desde Salmonella typhi Salmonella enteritidis. Rev Chil Infectol 2001; 18: 85-93.

8.- Departamento de Epidemiología. MINSAL, Chile. Informe brote listeriosis en la Región Metropolitana. 5 de febrero del 2009. Disponible en http://epi.minsal.cl/epi/html/ bolets/reportes/Listeriosis/Informe\%20 Listeria\%20brote\%202008.pdf (accedido en agosto de 2010).

9.- $\quad$ Noriega LM, Ibáñez S, González P, Yamamoto
M, Astudillo J, González M, et al. Listeria monocytogenes: informe de un aumento de casos en mujeres embarazadas y revisión de la literatura. Rev Chil Infectol 2008; 25: 342-9.

10.- Miranda G, Orellana P, Dellien H, Switt M. Romboencefalitis por Listeria monocytogenes. Patología emergente en relación al brote epidémico. Presentación de 3 casos clínicos. Rev Med Chile 2009; 137: 1602-6.

11.- Gazaigne L, Legrand P, Renaud B, Bourra B, Taillandier E, Brun-Buisson C, et al. Campylobacter fetus bloodstream infection: risk factors and clinical features. Eur J Clin Microbiol Infect Dis 2008; 27: 185-9.

12.- Nielsen, H, Hansen K K, Gradel KO, Kristensesn B, Ejlertsen T, C. Østergaard O, et al. Bacteraemia as a result of Campylobacter species: a population-based study of epidemiology and clinical risk factors. Clin Microbiol Infect 2010; 16: 57-61.

13.- Mead P S, Slutsker L, Dietz V, McCaig L F, Bresee J S, Shapiro C, et al. Food-related illness and deaths in the United States. Emerg Infect Dis 1999; 5: 607-25.

14.- Figueroa G, Troncoso M, López C, Rivas P, 
Toro M. Occurrence and enumeration of Campylobacter spp. during the processing of Chilean broilers. BMC Microbiology 2009; 9:94. doi:10.1186/1471-2180-9-94

15.- Tremblay C, Gaudreau C, Lorange M. Epidemiology and antimicrobial susceptibilities of 111 Campylobacter fetus subsp. fetus strains isolated in Québec, Canada, from 1983 to 2000. J Clin Microbiol 2003; 41: 463-6.

16.- Font C, Cruceta A, Moreno A, Miró O, Coll-Vinent B, Almela M, et al. Estudio de 30 pacientes con bacteriemia por Campylobacter spp. Med Clin (Barc) 1997; 108: 336-40.

17.- Nielsen H, Persson S, Olsen KEP, Ejlertsen T, Kristensen B, Schønheyder HC. Bacteraemia with Campylobacter jejuni: no association with the virulence genes iam, $c d t B$, capA or virB. Eur J Clin Microbiol Infect Dis 2010; 29: 357-8.
18.- García P, Valenzuela N, Rodríguez V, León E, Fernández H. Susceptibilidad antimicrobiana de Campylobacter jejuni aislado de coprocultivos en Santiago de Chile. Rev Chil Infectol 2009; 26: 511-14.

19.- Pacanowski J, Lalande V, Lacombe $\mathrm{K}$, Boudraa C, Lesprit P, Legrand P, et al. Campylobacter bacteremia: Clinical features and factors associated with fatal outcome. Clin Infect Dis 2008; 47: 790-6.

20.- Streit J M, Jones R N, Toleman M A, Stratchounski L S, Fritsche T R. Prevalence and antimicrobial susceptibility patterns among gastroenteritis-causing pathogens recovered in Europe and Latin America and Salmonella isolates recovered from bloodstream infections in North America and Latin America: Report from the SENTRY Antimicrobial Surveillance Program (2003). Int J Antimicrob Agents 2006;
27: 367-75.

21.- Tajada P, Gómez-Garcés J L, Alos J I, Balas D, Cogollos R. Antimicrobial susceptibilities of Campylobacter jejuni and Campylobacter coli to $12 \beta$-lactam agents and combination with $\beta$-lactamase inhibitors. Antimicrob Agents Chemother 1996; 40: 1924-5.

22.- Woo P C Y, Leung K W, Tsoi H W, Wong S S Y, Teng J L L, Yuen K Y. Thermo-tolerant Campylobacter fetus bacteraemia identified by $16 \mathrm{~S}$ ribosomal RNA gene sequencing: an emerging pathogen in immunocompromised patients. J Med Microbiol 2002; 51: 740-6.

23.- Prado V, Martínez J, Reyes L, Ducheylard M, Bercovich M, Millán V, et al. Características de la infección intestinal por Campylobacter jejuni en lactantes chilenos. Rev Med Chile 1985; 113: 521-25. 